Transcontinentales

continentales

Sociétés, idéologies, système mondial

$7 \mid 2009$

Varia

\title{
Maintenir le cap de Transcontinentales
}

\section{Dominique Fournier}

\section{OpenEdition}

\section{Journals}

Édition électronique

URL : http://journals.openedition.org/transcontinentales/811

DOI : 10.4000/transcontinentales.811

ISBN : 978-2-7351-1559-4

ISSN : 1775-397X

Éditeur

Editions de la maison des sciences de l'homme

Édition imprimée

Date de publication : 30 juin 2009

ISBN : 978-2-200-92512-3

ISSN : 1950-1684

Référence électronique

Dominique Fournier, « Maintenir le cap de Transcontinentales », Transcontinentales [En ligne], 7 | 2009, mis en ligne le 24 mars 2011, consulté le 25 septembre 2020. URL : http://journals.openedition.org/ transcontinentales/811; DOI : https://doi.org/10.4000/transcontinentales.811 


\section{Maintenir le cap de Transcontinentales}

Depuis le temps qu'ils se sont implantés à travers le monde, les instituts français de recherche à l'étranger (IFRE) n'ont jamais manqué l'occasion de répondre aux sollicitations scientifiques légitimes venues du pays qui acceptait de les accueillir ou à celles qui émanaient du milieu de la recherche française. Forts du principe de rotation de leurs chercheurs et convaincus de leur mission essentielle de formation de spécialistes tournés vers le futur, ils ont le plus souvent réussi leur insertion dans le paysage scientifique d'un monde en évolution en affirmant leur volonté d'apporter un témoignage et de contribuer à la diffusion d'une pensée originale d'autant plus féconde qu'elle reste en contact étroit avec les réalités des régions étudiées. Tous les instituts, sur quelque continent qu'ils se trouvent, ont également su faire preuve de leur volonté de s'adapter peu ou prou aux techniques d'information modernes qui leur permettent de faire connaître leur action et les résultats des travaux entrepris sous leur égide. L'usage de ces moyens de communication et une politique de publication adaptée ont permis à chacun d'entre eux de toucher au moins sa région et l'aire culturelle environnante, mais comme les instituts ont vocation à s'exprimer à plus large échelle, Transcontinentales, revue destinée à une communication globalisée, se doit de proposer un complément de haut niveau à leur politique de valorisation.

Depuis plusieurs années, la question se pose avec beaucoup de force de l'opportunité de poursuivre ou non la politique de diffusion de revues scientifiques au moyen de $l^{\prime}$ édition traditionnelle. Transcontinentales $\mathrm{n}^{\circ} 7$ devrait constituer le dernier numéro "papier» réalisé avec le soutien efficace de notre éditeur, et il bénéficiera des capacités de distribution habituelles. Dans la configuration actuelle des politiques d'échange de l'information, la possibilité de proposer notre revue en ligne doit être envisagée afin que les recherches en sciences sociales et humaines réalisées dans le cadre des instituts soient mieux appréhendées dès lors qu'il s'agit de témoigner à la fois de la variété des thèmes abordés, des méthodes utilisées et, d'une certaine façon, d'une manière d'approche théorique cohérente des problèmes spécifiques affectant des sociétés soumises à un mouvement de globalisation accéléré. Rendue plus accessible et plus largement diffusée, une revue telle que Transcontinentales doit pouvoir être le support privilégié du réseau des IFRE, un des modes d'expression d'une recherche d'excellence soutenue par le ministère des Affaires étrangères et européennes et, désormais, le CNRS; une recherche élaborée conjointement avec nos partenaires hôtes, faite d'approche fine du terrain, d'échanges à courte et longue 
distance. Dans cette nouvelle configuration définie par l'univers électronique, il est probable que le site www.ifre.fr s'imposera comme un outil de communication complémentaire en améliorant les capacités d'interactivité, de réactivité face à l'actualité. N'est-il pas important d'exposer de façon immédiate et sur un support à vocation généraliste des analyses initiales portant sur des faits fournis par une actualité de retentissement mondial? Transcontinentales électronique a besoin d'un pendant davantage tourné vers le quotidien des équipes et, mis en lien avec chacun des sites concernés, www.ifre.fr doit devenir une vitrine à multiples facettes où s'exprimerait plus largement encore toute la valeur de l'expertise de nos chercheurs.

Contrairement aux six premiers numéros thématiques qui, grâce à l'impulsion de Jean-Luc Racine, ont constitué un apport particulièrement remarquable à une réflexion portant sur certains aspects majeurs de la contemporanéité (le défi sanitaire, mémoire et nations, réforme et "grand Moyen-Orient»), ainsi que sur la situation géopolitique dans divers continents (Amérique latine, Afrique plurielle, la transition chinoise), ce numéro entend donner aux lecteurs une idée du spectre élargi des problématiques abordées par les équipes formées dans les instituts. Alors qu'ils étaient regroupés auparavant dans une simple rubrique, les varia représentent ici la trame même de la revue, témoignant d'une variété, d'une acuité et d'une originalité qu'un réseau de l'ampleur de celui des IFRE est forcément à même d'offrir à ceux que préoccupe une situation mondiale en perpétuelle mutation.

Il est ici question d'Afrique, d'Amérique latine, d'Asie, du Moyen-Orient, voire d'une définition polémique de l'Europe du Sud, mais si nous voulions déterminer un principe d'unité à ce bel ensemble, nous le trouverions du côté d'une participation résolue des différents acteurs évoqués à un monde en marche. Plusieurs articles évoquent des situations qui contraignent des hommes et des groupes à réaffirmer leur appartenance identitaire tout en s'efforçant de s'adapter du mieux possible à une vie nouvelle, un état de transition souvent. Le travail des chercheurs révèle la multiplicité des facteurs qui concourent au surgissement de crises plus ou moins récurrentes, plus ou moins violentes. Car face à l'attractivité d'une modernité forcée et de métropoles aux contours parfois incertains, il ne manque pas de réponses adaptatives pertinentes venues de communautés que d'aucuns s'entêtent à vouloir qualifier de rétrogrades. Qu'ils soient marqués par une religiosité profonde - les salafistes yéménites, les juifs éthiopiens en Israël -, ou motivés par leur attachement à un mode de vie campagnard traditionnel, les acteurs de ce monde mouvant ne sont pas prêts à abandonner sans comprendre la part la plus féconde de leur identité. Ils entendent évidemment que la vie quotidienne de leurs familles bénéficie elle aussi des apports majeurs des techniques élaborées au cours des dernières décennies pour améliorer l'accès aux ressources indispensables - l'eau par exemple -, mais ils se heurtent souvent, comme dans le cas paradigmatique du Kenya, à la réaction d'instances supérieures animées par d'autres logiques et d'autres valeurs.

Il reste que le monde moderne, soucieux parfois de préserver une stabilité sociale favorable à ceux qui en détiennent les clés, multiplie les méthodes destinées à affirmer leur ancrage dans la modernité, à améliorer la vie quotidienne de ceux qui se reconnaissent chaque jour davantage comme des citoyens, et à permettre 
l'insertion des nouveaux entrants - ce sont parfois des pays voisins - dans des systèmes tentés par une certaine forme de globalisation. Les articles consacrés à la politique culturelle à Istanbul et à l'assistance juridique japonaise montrent que les solutions apportées à certains problèmes ne s'adressent pas seulement à la population locale, mais qu'elles doivent être conçues en termes d'image internationale, et d'influence plus ou moins étendue. Comment imaginer que l'instabilité générée par ces confrontations intercommunautaires et internationales permanentes ne doive pas faire l'objet de l'établissement de règles susceptibles d'être tolérées par le plus grand nombre? L'article en forme de note finale consacré à ce que nous pourrions qualifier de retour du droit dans le giron des sciences humaines et sociales fournit en fait une piste. S'appuyant de façon polémique sur les avancées ou les frilosités apparues lors des débats ponctués par plusieurs colloques internationaux et une publication déjà ancienne du CNRS, il nous propose de réfléchir à la place qu'auront à occuper les IFRE dans l'inévitable mouvement de recherches qui va parcourir le monde lorsqu'il faudra analyser le problème de l'adéquation des théories juridiques, des normes internationales, avec la réalité des conditions socioculturelles propres à chaque pays.

Le futur Transcontinentales électronique sera là pour donner à connaître la place de notre droit et de ses évolutions dans le paysage international, comme il permettra dès les prochains numéros de publier les résultats du colloque du réseau des IFRE des 5 et 6 janvier 2010 consacré aux enjeux des grands courants migratoires actuels. Les IFRE possèdent des chercheurs de haut niveau capables de traiter non seulement des pays d'accueil, mais surtout des zones d'origine des migrants. Quel meilleur support que le nôtre pour informer sur l'originalité de nos analyses, et leur force de proposition? 